\title{
Frequency of phantom scratching in Cavalier King Charles Spaniels (CKCS) assessed by owner questionnaire before and during maropitant treatment
}

Emma Gilbert ${ }^{1}$, Sandra Sanchis Mora² ${ }^{2}$ Ludovic Pelligand², Lydia Poad², Holger Volk ${ }^{3}$ Clare Rusbridge ${ }^{4}$.

1. Lumbry Park Veterinary Specialists. 2. Clinical Science and services, Royal Veterinary College. 3. University of Veterinary Medicine Hannover, 4. Fitzpatrick Referrals Ltd and University of Surrey

Funding was provided by Cavalier Matters Charity. Please send any questions to emma.gilbert@cvsvets.com
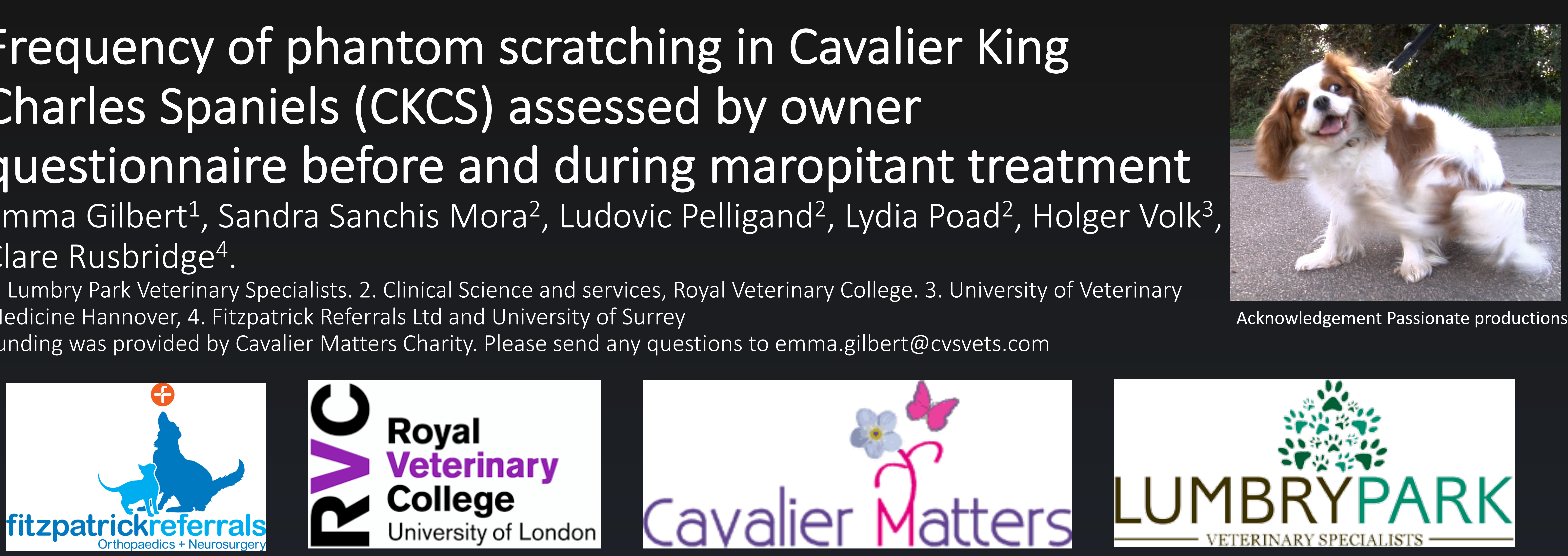

Study basis

- The superficial dorsal horn was previously found to be affected in CKCS with syringomyelia with the clinical sign of phantom scratching (1).

- The NK-1 receptors located in this area are considered as a target for treatment for fictive itch (2).

- We aimed to assess outcomes with 2 weeks maropitant treatment (NK-1 R antagonist) on frequency of phantom scratching.

- RVC Ethical approval URN: 2018 1835-3

- There were no conflicts of interest.

Study design:
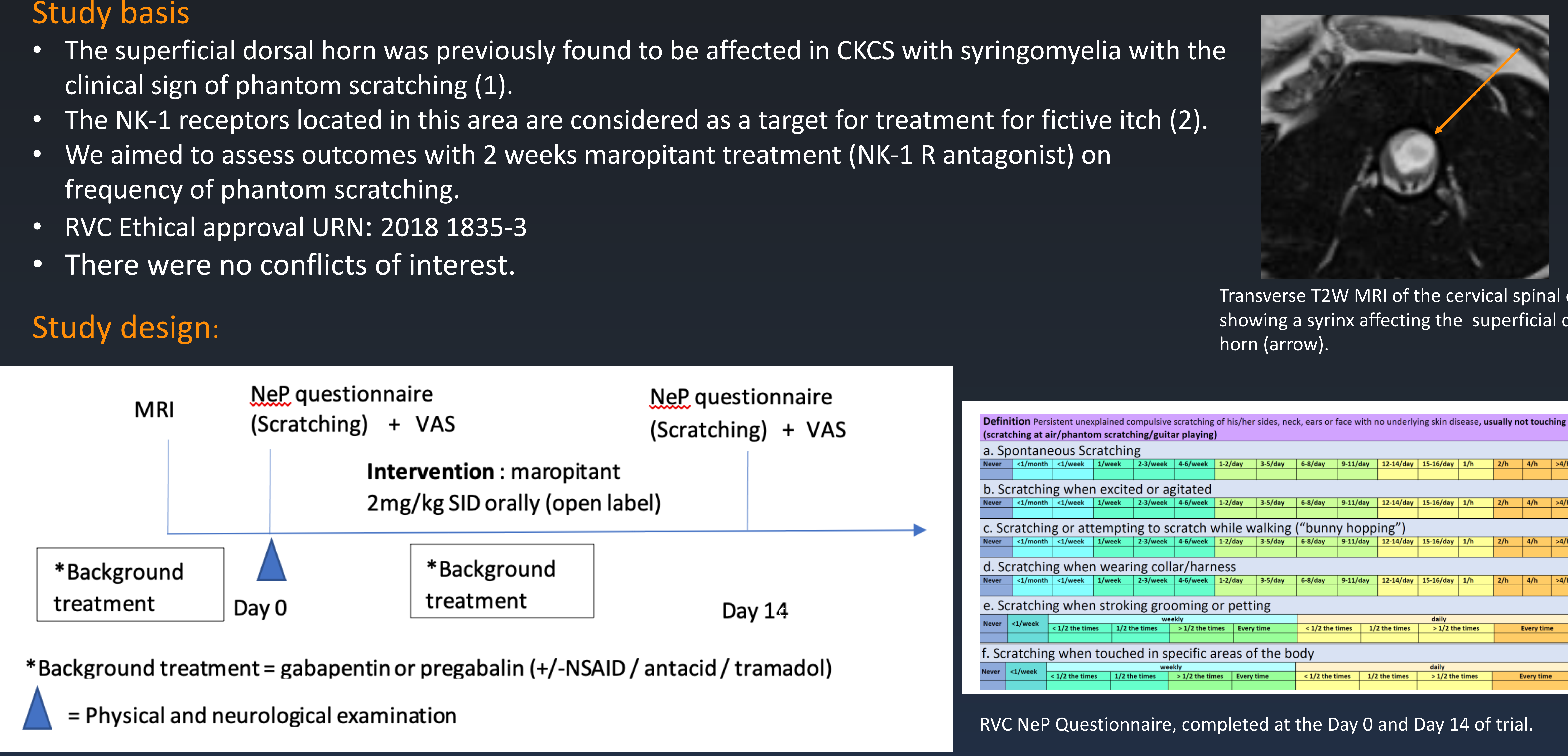

Transverse T2W MRI of the cervical spinal cord showing a syrinx affecting the superficial dorsal horn (arrow).

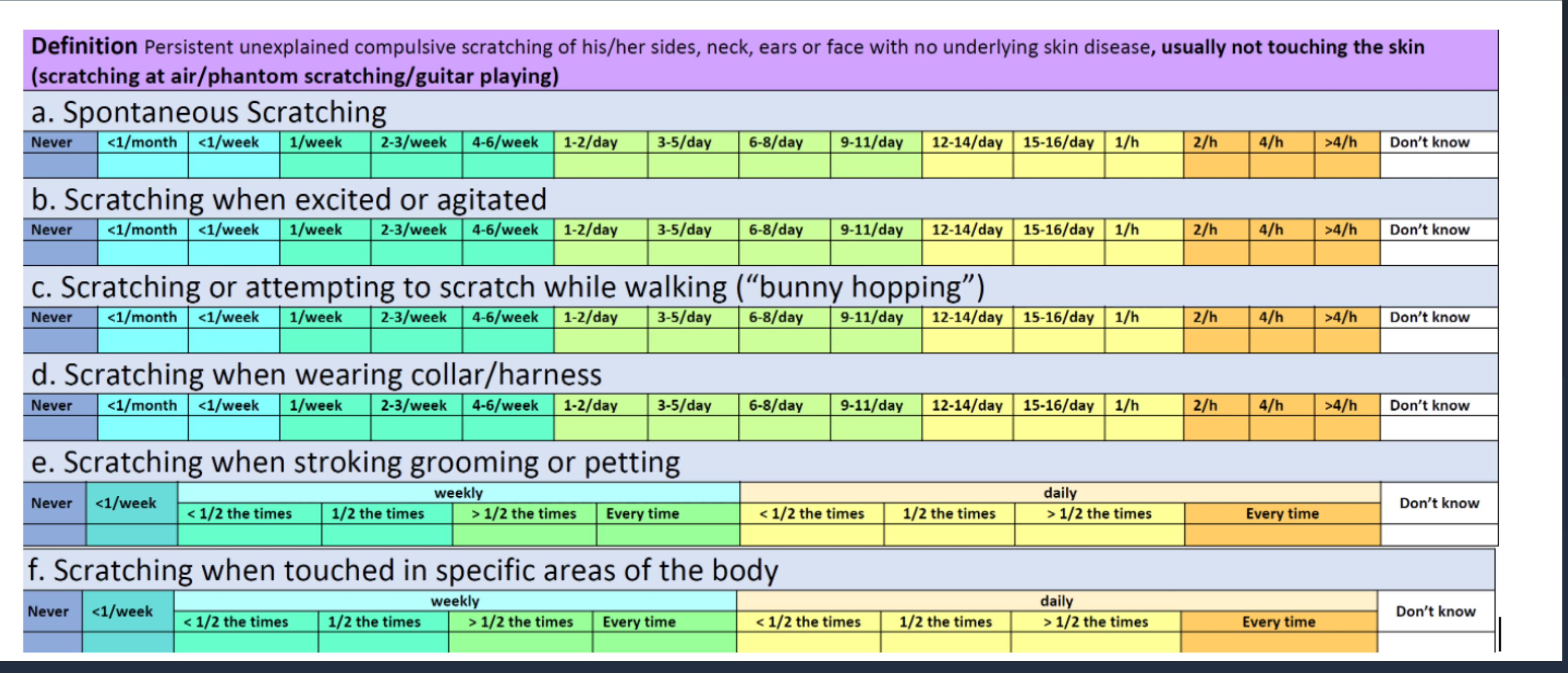

RVC NeP Questionnaire, completed at the Day 0 and Day 14 of trial.

Results:

- Population: 9 CKCS, mean age 6.1 years (range 3-10), 3 males ( 1 entire, 2 neutered) and 6 females (1 entire, 5 neutered).

- The most common site that the syrinx involved the superficial dorsal horn was the level of C3 or C4 vertebrae.

- Following treatment with maropitant mean scores for questions on frequency of phantom scratching decreased (Table below).

- Wilcoxon Rank sign analysis was significant in $4 / 6$ of these questions. Spontaneous scratching ( $p=0.017)$, scratching while walking ( $p=0.011)$, scratching when groomed $(p=0.017)$, scratching when touched $(p=0.026)$.

\begin{tabular}{l|l|l|l|l|l|l|}
\hline Scratch trigger & Spontaneous & Excitement or agitation & Walking & $\begin{array}{l}\text { Wearing Collar or } \\
\text { harness }\end{array}$ & $\begin{array}{l}\text { Stroking or } \\
\text { grooming }\end{array}$ & $\begin{array}{l}\text { Touch of specific } \\
\text { body area }\end{array}$ \\
\hline $\begin{array}{l}\text { Number of cases with } \\
\text { decreased/increased } \\
\text { frequency }\end{array}$ & $7 / 1$ & $5 / 1$ & $8 / 0$ & $4 / 1$ & $7 / 0$ & $6 / 0$ \\
$\begin{array}{l}\text { Mean change in } \\
\text { frequency score }\end{array}$ & -3.33 & -1.22 & -4 & -3 & -1.88 & -1.55 \\
\hline
\end{tabular}

Discussion:

- VAS scores were difficult for owners to complete without guidance with only $3 / 9$ being completed (though notably all giving a decreased score consistent with improvement), we therefore excluded them from the results of our study.

- This trial was open label, and therefore subject to caregiver bias, placebo effect and high owner expectation $(3,4)$.

- Owners were happy with maropitant as a treatment for chronic disease due to its low side effect profile.

- Further investigation with a placebo controlled crossover study is needed next to confirm this initial evidence for a positive effect to decrease frequency of phantom scratching in dogs and the lack adverse effects found with treatment with this licensed veterinary product. 